\title{
INTERNAL RESONANCES AND MODES INTERACTIONS IN NON-LINEAR VIBRATIONS OF VISCOELASTIC HETEROGENEOUS SOLIDS
}

\author{
Igor V. Andrianov ${ }^{1}$, Vladyslav V. Danishevskyy², Graham Rogerson ${ }^{3}$ \\ ${ }^{1}$ Institute of General Mechanics, RWTH Aachen University, Germany \\ ${ }^{2}$ Department of Structural Mechanics and Strength of Materials, \\ Prydniprovska State Academy of Civil Engineering and Architecture, Ukraine \\ ${ }^{3}$ School of Computing and Mathematics, Keele University, UK
}

\begin{abstract}
The aim of the paper is to study how the viscous damping influences on modes coupling in non-linear vibrations of microstructured solids. As an illustrative example, natural longitudinal vibrations of a layered heterogeneous medium are considered. The macroscopic dynamic equation is obtained by the asymptotic homogenisation method. The input continuous problem is analysed using a spatial discretisation procedure. An asymptotic solution is developed by the method of multiple time scales and the fourth-order Runge-Kutta method is employed for numerical simulations. Internal resonances and energy transfers between the vibrating modes are predicted and analysed. The conditions for a possible truncation of the original infinite system are discussed. The obtained numerical and analytical results are in good agreement.
\end{abstract}

Key words: non-linear vibrations; internal resonances; modes coupling; dissipation; microstructure; homogenisation; multiple time scales.

\section{Introduction}

Real materials and structures are never perfectly elastic. In dynamic problems, a part of the mechanical energy is always transformed to heat because of the internal friction. Dissipative behaviour of heterogeneous solids can be determined by various factors, such as properties of the components, features of the microstructure, bonding conditions at the "matrix-inclusion" interface, etc. [1-4]. Here we address the case when energy dissipation is caused by the viscoelasticity of the components, which is typical, for example, for polymer-based materials.

Viscoelastic properties of the medium can be described by the well known Kelvin-Voigt model (see, for example, [5]). In hydrodynamics it corresponds to the classical behaviour of a viscous gas, where shear stresses are proportional to the deformation rates and the proportionality coefficients are determined by the gas density. For solids, the Kelvin-Voigt model can be naturally 
deduced from a lattice-type model passing to the continuous limit and assuming that the interaction forces between neighbouring particles depend on the rate of the distance change rather than on the distance itself [4].

Pal'mov [6,7] studied non-linear vibrations of semi-infinite and finite rods subjected to dissipation. He employed the method of harmonic linearisation, which gave the first order approximation coinciding with the Rayleigh-Ritz approach. Equations in slow variables were obtained and analysed. High-frequency deformations of non-linear rate dependent materials induced by the propagation of transient waves were considered by Varley and Rogers [8] and Seymour and Varley [9]. Mortell and Varley [10] discussed non-linear elastic waves in finite-size bodies and studied the dynamic response of a viscoelastic rod subjected to a pulse load [11]. In the first order approximation the solution was developed as a superposition of two modulated waves travelling into the opposite directions and not interacting with each other.

Vibrations of continuous structures can be described by dynamical systems having infinite degrees of freedom. Non-linearity leads to localisation of energy and its transfer from the low- to the high-frequency part of the spectrum and vice versa. Then the vibration modes can be involved into complicated interactions, which result in internal resonances and in a self-generation of higherorder modes. In such a case, truncation to the modes having non-zero initial energy (which is usually applicable in linear problems) is not possible and all the resonant modes should be taken into account.

The effects of modes coupling and internal resonances have attracted a considerable attention of many authors. A number of results were obtained for vibrations of homogeneous structures and numerical [12] as well as asymptotic [13-17] approaches have been widely applied. However, the non-linear dynamic behaviour of heterogeneous solids was studied to a significantly less extent. Only recently, one-dimensional vibrations of microstructured rods were considered by Andrianov et al. with an account to a non-linear elastic external medium [18] and to geometrical and physical non-linearity [19]. Heterogeneity results in dispersion of energy and, thus, compensates the influence of non-linearity. As the spatial period of the modes decreases and approaches the size of the microstructure, the internal resonances are suppressed and a truncation to only a few leading order modes can be justified.

In our previous works we omitted the influence of viscous damping. It should be noted that dissipation restrains energy transfers between the vibration modes and on a large time scale the modes coupling vanishes. In that sense dispersion and dissipation acting in a non-linear system may lead to qualitatively similar physical consequences (Zabusky and Kruskal encountered this analogy analysing the FPU problem, see [20]). 
In this paper, non-linear vibrations of a layered viscoelastic solid are studied. We propose a macroscopic wave equation describing the dynamic behaviour of the body with an account to the properties of the microstructure. Basing on the developed model, the interplay between the effects of non-linearity and dissipation is analysed. We aim to predict how the viscous damping influences on the modes coupling and to justify a truncation of the original infinite system to a finite number of the leading order modes.

The paper is organised as follows. In Section 2, the input constitutive relations are introduced and the homogenised dynamical equation is formulated. In Section 3, the discretisation procedure is presented and two different types of the mode interactions are discussed. In Section 4, natural vibrations are studied by the method of multiple time scales. Section 5 is devoted to the numerical simulation of the modes coupling and to a comparison of the numerical and asymptotic solutions. Concluding remarks are presented in Section 6.

\section{Input problem and homogenised dynamical equation}

Let us consider a heterogeneous solid consisting of periodically repeated viscoelastic layers $\Omega^{(1)}$ and $\Omega^{(2)}$ (Fig. 1). We shall study natural longitudinal vibrations in the direction $x$. This model can describe the properties of laminated composite materials, phononic crystals, acoustic diodes (see, for example, [21-23]). Layered structures are employed in band-gap engineering for the design of vibration and sound control devices [24].

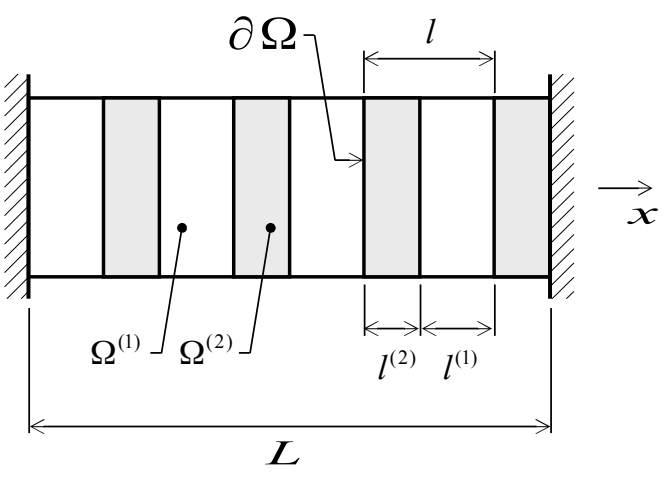

Fig. 1. Periodically heterogeneous solid under consideration.

The mechanical behaviour of each of the layers $\Omega^{(i)}$ is described by the Kelvin-Voigt model [5], which includes a purely elastic spring connected in parallel with a viscous dashpot. The properties of the dashpot are assumed to be linear, while the spring exhibits a non-linear response. Geometric non-linearity is taken into account using the Cauchy-Green strain tensor [25] and 
physical non-linearity is modelled with the help of the Murnaghan elastic potential [26]. The time dependent longitudinal stress $\sigma^{(i)}$ is given by

$$
\sigma^{(i)}=\alpha^{(i)} \frac{\partial u^{(i)}}{\partial x}+\frac{\beta^{(i)}}{2}\left(\frac{\partial u^{(i)}}{\partial x}\right)^{2}+\eta^{(i)} \frac{\partial^{2} u^{(i)}}{\partial x \partial t}
$$

where $u^{(i)}$ is the displacement; $\alpha^{(i)}$ and $\beta^{(i)}$ are the elastic coefficients; $\alpha^{(i)}=\lambda^{(i)}+2 \mu^{(i)}, \lambda^{(i)}, \mu^{(i)}$ are the Lamé elastic constants; $\beta^{(i)}=3\left(\lambda^{(i)}+2 \mu^{(i)}\right)+2\left(A^{(i)}+3 B^{(i)}+C^{(i)}\right), A^{(i)}, B^{(i)}, C^{(i)}$ are the Landau elastic constants; $\eta^{(i)}$ is the viscosity. Here and in the sequel the upper indexes $(i), i=1,2$, refer to the different components $\Omega^{(1)}, \Omega^{(2)}$ of the medium.

The governing dynamical equation reads

$$
\left(\alpha^{(i)}+\eta^{(i)} \frac{\partial}{\partial t}\right) \frac{\partial^{2} u^{(i)}}{\partial x^{2}}+\beta^{(i)} \frac{\partial u^{(i)}}{\partial x} \frac{\partial^{2} u^{(i)}}{\partial x^{2}}=\rho^{(i)} \frac{\partial^{2} u^{(i)}}{\partial t^{2}},
$$

where $\rho^{(i)}$ is the mass density. At the interface $\partial \Omega$, we assume the conditions of perfect bonding that imply the continuity of the displacement and stress fields:

$$
u^{(1)}=u^{(2)}, \quad \sigma^{(1)}=\sigma^{(2)} \text { at } \partial \Omega .
$$

Let us suppose the characteristic size $l$ of the microstructure to be essentially smaller than the spatial period $L_{n}$ of a considered vibration mode. Then the input problem (2.1), (2.2) can be replaced by a homogenised dynamical equation

$$
\left(\langle\alpha\rangle+\langle\eta\rangle \frac{\partial}{\partial t}\right) \frac{\partial^{2} u}{\partial x^{2}}+\langle\beta\rangle \frac{\partial u}{\partial x} \frac{\partial^{2} u}{\partial x^{2}}=\langle\rho\rangle \frac{\partial^{2} u}{\partial t^{2}}
$$

where $u$ is the macroscopic displacement field; $\langle\cdot\rangle$ denotes the homogenised (so called effective) properties of the medium. Equation (2.3) presents the leading order $O\left(\delta^{0}\right)$ approximation, where $\delta=l / L_{n} \ll 1$ is the natural small parameter. We note that taking into account higher-order terms in powers of $\delta$ will lead to additional high-gradient terms in equation (2.3), which describe dispersion of the vibration energy by the microstructure. Higher-order homogenised models have been 
considered in our previous works $[18,19]$. In this paper we focus on the study of dissipation and, for the sake of clarity, specialise the analysis to the $O\left(\delta^{0}\right)$ approximation only.

The effective mass density $\langle\rho\rangle$ is given by a simple rule of mixture: $\langle\rho\rangle=c^{(1)} \rho^{(1)}+c^{(2)} \rho^{(2)}$, where $c^{(i)}$ are the volume fractions of the components, $c^{(i)}=l^{(i)} / l$.

In the first approximation, the elastic and the viscous properties can be homogenised independently of each other. This simplified procedure may provide a reasonable approach if nonlinearity and viscosity are small. The effective elastic coefficients $\langle\alpha\rangle$ and $\langle\beta\rangle$ are evaluated from the rate independent problem [27] as follows:

$$
\begin{gathered}
\langle\alpha\rangle=\frac{\alpha^{(1)} \alpha^{(2)}}{c^{(1)} \alpha^{(2)}+c^{(2)} \alpha^{(1)},} \\
\langle\beta\rangle=\frac{c^{(1)} \beta^{(1)}\left(\alpha^{(2)}\right)^{3}+c^{(2)} \beta^{(2)}\left(\alpha^{(1)}\right)^{3}}{\left(c^{(1)} \alpha^{(2)}+c^{(2)} \alpha^{(1)}\right)^{3}} .
\end{gathered}
$$

In order to determine the effective viscosity $\langle\eta\rangle$, let us apply to equations (2.1), (2.2) the Laplace transform $u_{s}^{(i)}(x, s)=\int_{0}^{\infty} u^{(i)}(x, t) \exp (-s t) d t$. Assuming zero initial conditions, in the linear approximation we obtain

$$
\begin{gathered}
\left(\alpha^{(i)}+s \eta^{(i)}\right) \frac{\partial^{2} u_{s}^{(i)}}{\partial x^{2}}=s^{2} \rho^{(i)} u_{s}^{(i)} \\
u_{s}^{(1)}=u_{s}^{(2)}, \quad\left(\alpha^{(1)}+s \eta^{(1)}\right) \frac{\partial u_{s}^{(1)}}{\partial x}=\left(\alpha^{(2)}+s \eta^{(2)}\right) \frac{\partial u_{s}^{(2)}}{\partial x} \text { at } \partial \Omega .
\end{gathered}
$$

Homogenisation of the problem (2.6), (2.7) gives [28]

$$
\langle\alpha+s \eta\rangle \frac{\partial^{2} u_{s}}{\partial x^{2}}=s^{2}\langle\rho\rangle u_{s}^{(i)},
$$

where $\langle\alpha+s \eta\rangle=\left[c^{(1)} /\left(\alpha^{(1)}+s \eta^{(1)}\right)+c^{(2)} /\left(\alpha^{(2)}+s \eta^{(2)}\right)\right]^{-1}$

Application of the inverse Laplace transform leads to an integro-differential equation, which complicates significantly the further analysis. To avoid this, in the case of small viscosity we represent the homogenised parameter $\langle\alpha+s \eta\rangle$ as a power series expansion 


$$
\langle\alpha+s \eta\rangle=\frac{\alpha^{(1)} \alpha^{(2)}}{c^{(1)} \alpha^{(2)}+c^{(2)} \alpha^{(1)}}+s \frac{c^{(1)} \eta^{(1)}\left(\alpha^{(2)}\right)^{2}+c^{(2)} \eta^{(2)}\left(\alpha^{(1)}\right)^{2}}{\left(c^{(1)} \alpha^{(2)}+c^{(2)} \alpha^{(1)}\right)^{2}}+O\left(s^{2}\right) .
$$

Substituting (2.7) into (2.6) and making the inverse transform, one obtains the linear part of the homogenised equation (2.3), where the effective elastic coefficient $\langle\alpha\rangle$ is given by formula (2.4) and the effective viscosity $\langle\eta\rangle$ is obtained as follows

$$
\langle\eta\rangle=\frac{c^{(1)} \eta^{(1)}\left(\alpha^{(2)}\right)^{2}+c^{(2)} \eta^{(2)}\left(\alpha^{(1)}\right)^{2}}{\left(c^{(1)} \alpha^{(2)}+c^{(2)} \alpha^{(1)}\right)^{2}} .
$$

Equation (2.3) describes the dynamic behaviour of the heterogeneous solid on the macro scale associated with the spatial period of the vibration modes. We note that all the effective coefficients are determined explicitly in terms of the microscopic properties of the medium. This contrasts with many well-known phenomenological approaches (e.g., Cosserat and Biot's theories), where the material parameters are usually left undetermined.

\section{Discretisation procedure}

Let us introduce non-dimensional variables $\bar{x}=x(\pi / L), \bar{t}=t(\pi / L) \sqrt{\langle\alpha\rangle /\langle\rho\rangle}, \bar{u}=u / A$, where $L$ is the length of the entire heterogeneous solid and $A$ is the vibration amplitude. For the simplicity, the over bars are omitted in the sequel. Equation (2.3) reads

$$
\frac{\partial^{2} u}{\partial x^{2}}+\gamma \frac{\partial^{3} u}{\partial x^{2} \partial t}+\varepsilon \frac{\partial u}{\partial x} \frac{\partial^{2} u}{\partial x^{2}}=\frac{\partial^{2} u}{\partial t^{2}}
$$

where $\gamma=\pi\langle\eta\rangle /(L \sqrt{\langle\alpha\rangle\langle\rho\rangle}), \quad \varepsilon=\pi A\langle\beta\rangle /(L\langle\alpha\rangle)$ are the non-dimensional small parameters characterising viscosity and non-linearity. The coefficient $\gamma$ is always positive, whereas the sign of $\varepsilon$ depends on the physical properties of the medium. For the most of materials (e.g., metals, polymers, stones) $\langle\beta\rangle<0$ and $\varepsilon<0$, which results in a soft non-linearity. A hard non-linearity, $\langle\beta\rangle>0$ , $\varepsilon>0$, can be observed for elastomers and rubber-like materials.

We consider a case of clamped-clamped edges. The boundary conditions are

$$
u(0, t)=u(\pi, t)=0 .
$$


In order to solve the boundary value problem (3.1), (3.2), a discretisation procedure is applied. Satisfying conditions (3.2), the displacement field is represented as a Fourier-sine expansion

$$
u(x, t)=q_{1}(t) \sin (x)+q_{2}(t) \sin (2 x)+q_{3}(t) \sin (3 x)+\ldots
$$

Substituting Ansatz (3.3) into equation (3.1), we come to an infinite system of non-linear ordinary differential equations

$$
\begin{gathered}
\frac{d^{2} q_{1}}{d t^{2}}+\gamma \frac{d q_{1}}{d t}+q_{1}+\varepsilon\left(q_{1} q_{2}+3 q_{2} q_{3}+6 q_{3} q_{4}+\ldots\right)=0, \\
\frac{d^{2} q_{2}}{d t^{2}}+4 \gamma \frac{d q_{2}}{d t}+4 q_{2}+\varepsilon\left(\frac{q_{1}^{2}}{2}+3 q_{1} q_{3}+8 q_{2} q_{4}+\ldots\right)=0, \\
\frac{d^{2} q_{3}}{d t^{2}}+9 \gamma \frac{d q_{3}}{d t}+9 q_{3}+\varepsilon\left(3 q_{1} q_{2}+6 q_{1} q_{4}+\ldots\right)=0, \\
\frac{d^{2} q_{4}}{d t^{2}}+16 \gamma \frac{d q_{4}}{d t}+16 q_{4}+\varepsilon\left(6 q_{1} q_{3}+4 q_{2}^{2}+\ldots\right)=0,
\end{gathered}
$$

which in a general form reads

$$
\frac{d^{2} q_{n}}{d t^{2}}+n^{2} \gamma \frac{d q_{n}}{d t}+n^{2} q_{n}+\frac{\varepsilon}{2} \sum_{m=1}^{\infty} m^{2} q_{m}\left[|m-n| q_{|m-n|}-(m+n) q_{m+n}\right]=0
$$

where $n=1,2,3, \ldots$

The obtained non-linear system is non-symmetric with respect to the odd and even vibration modes. A simple analysis reveals two fundamentally different types of the mode interactions as it was described by Chechin and Sakhnenko [29]. An initial excitation of the odd modes leads necessarily to the appearance of the even modes, i.e., equations (3.4) do not permit the solution $q_{2 n-1} \neq 0, q_{2 n}=0$. The even modes undergo a so called "force" excitation. On the other hand, the solution $q_{2 n-1}=0, q_{2 n} \neq 0$ is allowed and, in general, the presence of the even modes does not give rise to the odd modes. However, vibrations by the even modes only appear to be unstable and a small perturbation of the initial conditions induces energy transfers to the odd modes [19]. In this case the odd modes are subjected to a "parametric" excitation. 


\section{Method of multiple time scales}

We seek an asymptotic solution supposing non-linearity to be small, $|\varepsilon| \ll 1$. Let us introduce different time scales described by new independent variables $t_{0}=t, t_{1}=\varepsilon t, \ldots$, and represent $q_{n}$ as an expansion in powers of $\varepsilon$ :

$$
q_{n}(t)=q_{n 0}\left(t_{0}, t_{1}\right)+\varepsilon q_{n 1}\left(t_{0}, t_{1}\right)+O\left(\varepsilon^{2}\right) .
$$

The number of the required time scales depends on the order of approximation. We carry out expansion (4.1) to $O\left(\varepsilon^{2}\right)$, therefore, two time scales ("fast" $t_{0}$ and "slow" $t_{1}$ ) are needed. The derivatives with respect to $t$ are represented as follows

$$
\frac{d}{d t}=\frac{\partial}{\partial t_{0}}+\varepsilon \frac{\partial}{\partial t_{1}}+O\left(\varepsilon^{2}\right), \frac{d^{2}}{d t^{2}}=\frac{\partial^{2}}{\partial t_{0}^{2}}+2 \varepsilon \frac{\partial^{2}}{\partial t_{0} \partial t_{1}}+O\left(\varepsilon^{2}\right)
$$

Splitting system (3.4) in powers of $\varepsilon$, in $O\left(\varepsilon^{0}\right)$ approximation we obtain

$$
\frac{\partial^{2} q_{n 0}}{\partial t_{0}^{2}}+n^{2} \gamma \frac{\partial q_{n 0}}{\partial t_{0}}+n^{2} q_{n 0}=0
$$

The solution of equation (4.2) is

$$
q_{n 0}=\exp \left(-n^{2} \gamma t_{0} / 2\right)\left[a_{n}\left(t_{1}\right) \cos \left(\omega_{n} t_{0}\right)+b_{n}\left(t_{1}\right) \sin \left(\omega_{n} t_{0}\right)\right]
$$

where $\omega_{n}$ is the natural frequency of the $n$th mode in the linear case, $\omega_{n}^{2}=n^{2}\left(1-n^{2} \gamma^{2} / 4\right) ; a_{n}$ and $b_{n}$ are slow-varying amplitudes.

In $O\left(\varepsilon^{1}\right)$ approximation system (3.4) gives:

$$
\frac{\partial^{2} q_{n 1}}{\partial t_{0}^{2}}+n^{2} \gamma \frac{\partial q_{n 1}}{\partial t_{0}}+n^{2} q_{n 1}=-2 \frac{\partial^{2} q_{n 0}}{\partial t_{0} \partial t_{1}}-\frac{\varepsilon}{2} \sum_{m=1}^{\infty} m^{2} q_{m 0}\left[|m-n| q_{|m-n| 0}-(m+n) q_{m+n 0}\right] .
$$


A straightforward integration of system (4.4) will induce secular terms in the expressions for $q_{n 1}$. Secular terms grow without a bound in time, which is inconsistent with the physical properties of the conservative system under consideration. In order to eliminate secular terms, the coefficients of $\cos \left(\omega_{n} t_{0}\right)$ and $\sin \left(\omega_{n} t_{0}\right)$ in the r.h.s. of equations (4.4) must equal zero. Substituting (4.3) into (4.4) and expanding the products $q_{j 0} q_{k 0}$ using the standard trigonometric identities, we obtain a system of equations for $a_{n}$ and $b_{n}$, which gives a possibility to investigate the interactions between different modes.

In the non-dissipative case, $\gamma=0$, the frequencies $\omega_{n}$ become proportional with each other

$$
\omega_{j} / \omega_{k}=j / k
$$

Then an infinite number of modes are coupled by internal resonances. The presence of viscosity leads to a "detuning" of relation (4.5) and, on a large time scale, prevents energy transfers between the vibration modes.

Let us suppose viscosity to be small, $\gamma \ll 1$, and represent $\cos \left(\omega_{n} t_{0}\right)$ and $\sin \left(\omega_{n} t_{0}\right)$ in expression (4.3) as follows

$$
\begin{aligned}
& \cos \left(\omega_{n} t_{0}\right)=\cos \left(n t_{0}\right)+\frac{n^{3} \gamma^{2} t_{0}}{8} \sin \left(n t_{0}\right)+O\left(n^{6} \gamma^{4} t_{0}^{2}\right), \\
& \sin \left(\omega_{n} t_{0}\right)=\sin \left(n t_{0}\right)-\frac{n^{3} \gamma^{2} t_{0}}{8} \cos \left(n t_{0}\right)+O\left(n^{6} \gamma^{4} t_{0}^{2}\right) .
\end{aligned}
$$

Truncating expansions (4.6) to the leading order terms, we hold strictly the resonance condition (4.5). The physical sense of this approximation is that viscosity is considered to be responsible for energy dissipation (i.e., for exponential attenuation of $q_{n 0}$ ), but its influence on the natural frequencies is neglected and, thus, the internal resonances are not violated. The developed approach is applicable on a short time scale as $n^{3} \gamma^{2} t_{0} / 8 \ll 1$.

As it follows from (4.3), the attenuation coefficient of the $n$th mode is proportional to $n^{2}$. We observe that the internal dissipation exhibits a quadratic growth with the increase of the mode number and, therefore, the lowest modes can be the most important in vibrations of real structures. Let us examine resonant interactions between the modes 1 and 2 . It is convenient to introduce the amplitude $r_{n}\left(t_{1}\right)$ and the phase $\varphi_{n}\left(t_{1}\right)$ as follows: $a_{n}=r_{n} \cos \left(\varphi_{n}\right), b_{n}=r_{n} \sin \left(\varphi_{n}\right)$. Keeping the first two equations of system (4.4), the condition of elimination of secular terms gives: 


$$
\begin{gathered}
\frac{d r_{1}}{d t_{1}}=\frac{1}{4} r_{1} r_{2} \exp \left(-2 \gamma t_{0}\right) \sin \left(\varphi_{2}-2 \varphi_{1}\right), \\
r_{1} \frac{d \varphi_{1}}{d t_{1}}=-\frac{1}{4} r_{1} r_{2} \exp \left(-2 \gamma t_{0}\right) \cos \left(\varphi_{2}-2 \varphi_{1}\right), \\
\frac{d r_{2}}{d t_{1}}=-\frac{1}{16} r_{1}^{2} \exp \left(\gamma t_{0}\right) \sin \left(\varphi_{2}-2 \varphi_{1}\right), \\
r_{2} \frac{d \varphi_{2}}{d t_{1}}=-\frac{1}{16} r_{1}^{2} \exp \left(\gamma t_{0}\right) \cos \left(\varphi_{2}-2 \varphi_{1}\right) .
\end{gathered}
$$

Solution of system (4.7) in the purely elastic case $\gamma=0$ was analysed by Andrianov et al. [19]. We note that the vibration by the single mode 1 is not possible. If all the initial energy is accumulated in the mode 1 , i.e. $r_{1}(0) \neq 0, r_{2}(0)=0$, then the mode 2 is necessarily excited and a periodic energy exchange between the modes takes place. On the other hand, if we start with zero initial energy in the mode $1, r_{1}(0)=0, r_{2}(0) \neq 0$, then $d r_{2} / d t_{1}=d \varphi_{2} / d t_{1}=0$. The amplitude $r_{2}$ and the phase $\varphi_{2}$ are constant in time, so the system can vibrate by the mode 2 only. However, this regime is not stable in the sense that even a small perturbation of the initial conditions will induce the excitation of the mode 1 . The described behaviour is illustrated in the phase plane $\left(r_{2}, \varphi_{2}-2 \varphi_{1}\right)$ (see Fig. 2 in [19]).

\section{Numerical simulation of the modes coupling}

The distribution of energy among the vibration modes can be determined using Lagrangian formalism. Let us introduce a generalised equation of motion for the $n$th mode as follows

$$
\frac{d}{d t}\left[\frac{\partial T}{\partial\left(d q_{n} / d t\right)}\right]-\frac{\partial T}{\partial q_{n}}=-\frac{\partial V}{\partial q_{n}}-n^{2} \gamma \frac{d q_{n}}{d t},
$$

where $T$ is the kinetic and $V$ is the potential energy of the whole system. Integrating equations (3.4), we obtain

$$
T=\frac{1}{2} \sum_{n=1}^{\infty}\left(\frac{\partial q_{n}}{\partial t}\right)^{2}
$$




$$
V=\frac{1}{2} \sum_{n=1}^{\infty}\left\{n^{2} q_{n}^{2}+\varepsilon \int \sum_{m=1}^{\infty} m^{2} q_{m}\left[|m-n| q_{|m-n|}-(m+n) q_{m+n}\right] d q_{n}\right\}
$$

The term $\left(\partial q_{n} / \partial t\right)^{2}$ in expression (5.2) and the term $n^{2} q_{n}^{2}$ in expression (5.3) contribute to the $n$th equation of system (3.4). Then, in the leading order approximation and omitting the coefficient $1 / 2$, the total energy $E_{n}$ of the $n$th mode can be evaluated as follows

$$
E_{n}=\left(\frac{d q_{n}}{d t}\right)^{2}+n^{2} q_{n}^{2}+O(\varepsilon) .
$$

The second term in expression (5.3) represents a correction of the order $O(\varepsilon)$ to the potential energy $V$. It can include products $q_{m} q_{|m-n|}$ and $q_{m} q_{m+n}$ and, thus, its contribution is shared between the modes $m,|m-n|$ and $m+n$. Below we are restricted by the $O\left(\varepsilon^{0}\right)$ approximation and calculate the energy by formula (5.4).

The numerical examples are presented for the case of soft non-linearity, which is typical for the most industrial materials, and $\varepsilon=-0.1$. The increase in the magnitude of $\varepsilon$ makes the energy transfers faster, however, the qualitative behaviour of the solution remains the same. Numerical integration is performed by the Runge-Kutta fourth-order method in the open-source CAS Maxima. Practical convergence is verified by decreasing twice the step of the integration and checking that this does not affect the obtained numerical data.

Firstly, let us consider a two-mode approximation and compare the developed asymptotic approach (4.7) with the direct numerical integration of the two leading equations of the non-linear system (3.4). The initial energy is assumed to be localised in the mode 1. System (4.7) has a singularity at $r_{2}=0$; to avoid this the initial conditions are accepted as follows

$$
r_{2}=q_{2}=0.01, \quad r_{1}=q_{1}=\sqrt{1-4 r_{1}^{2}}=0.99979998, \quad \frac{d r_{1}}{d t}=\frac{d q_{1}}{d t}=\frac{d r_{2}}{d t}=\frac{d q_{1}}{d t}=0 \quad \text { at } \quad t=0 .
$$

Fig. 2 displays a periodic energy exchange between the modes 1 and 2. The asymptotic approximation of the method of multiple time scales is formally applicable at a relatively short time scale $t \sim O\left(\varepsilon^{-1}\right)$. However, it is numerically useful far beyond their nominal range of validity.

Next, let us examine interactions between the six leading modes. The initial conditions correspond to the case when all the input energy is localised in the mode 1 : 


$$
q_{1}=1, \quad q_{k}=0, \quad k=\overline{2,6} ; \quad \frac{d q_{n}}{d t}=0, \quad n=\overline{1,6} \text { at } t=0 .
$$

Fig. 3 presents the results of the numerical integration of system (3.4) truncated with $n=\overline{1,6}$. We observe that the increase in dissipation suppresses energy transfers and makes it possible to truncate the original infinite system to only a few leading order modes.

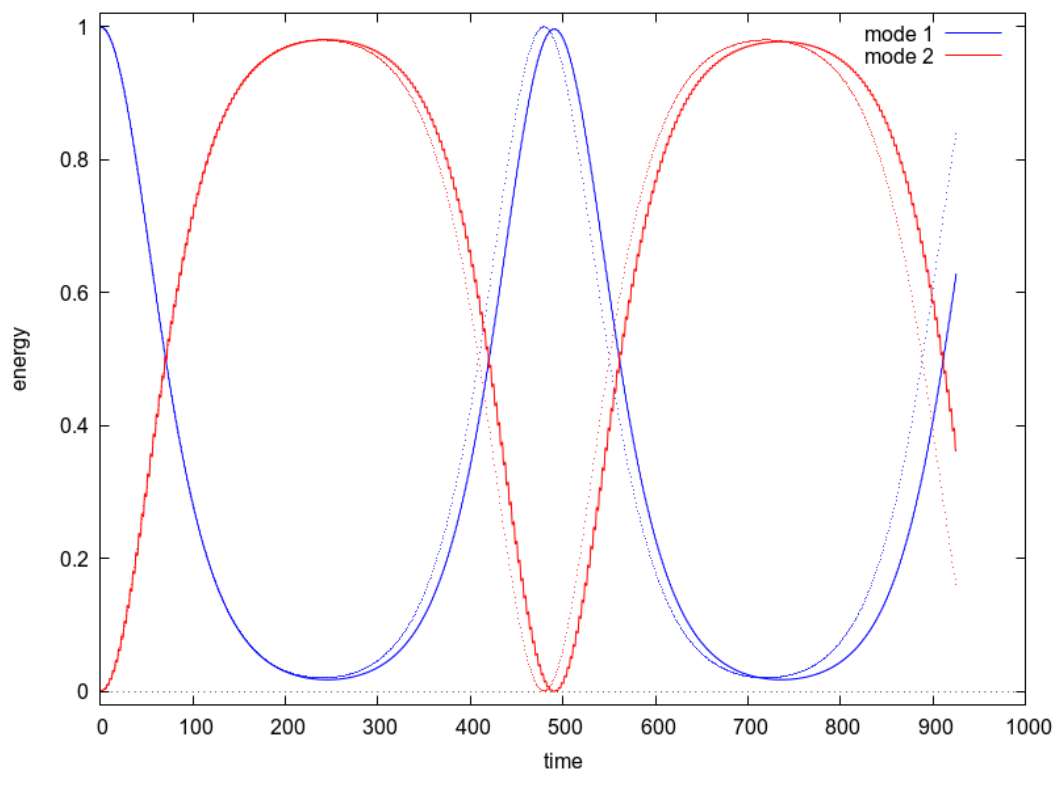

a) $\gamma=0$ (non-dissipative case)

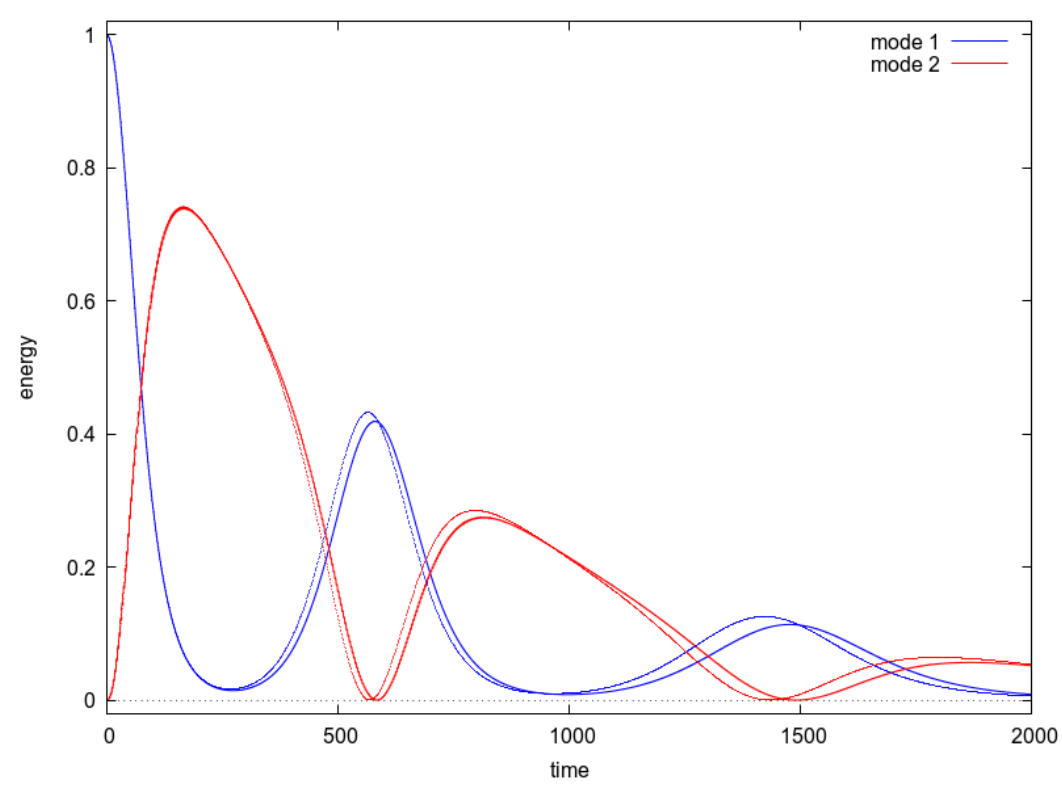

b) $\gamma=0.0005$ 


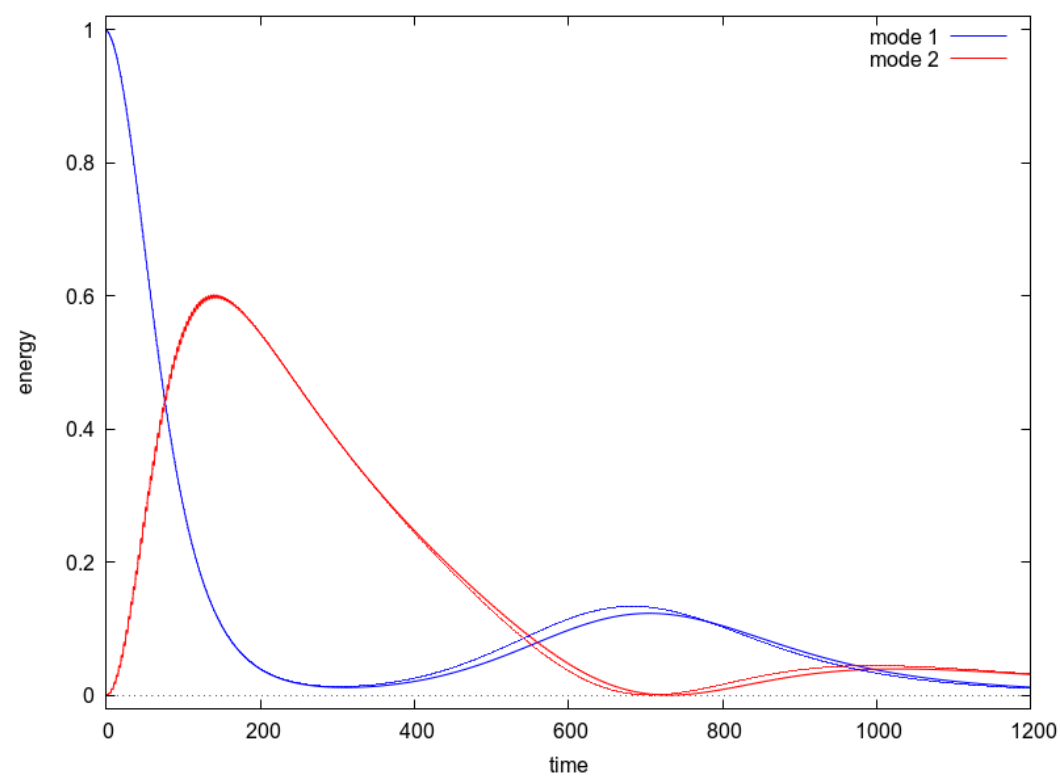

c) $\gamma=0.001$

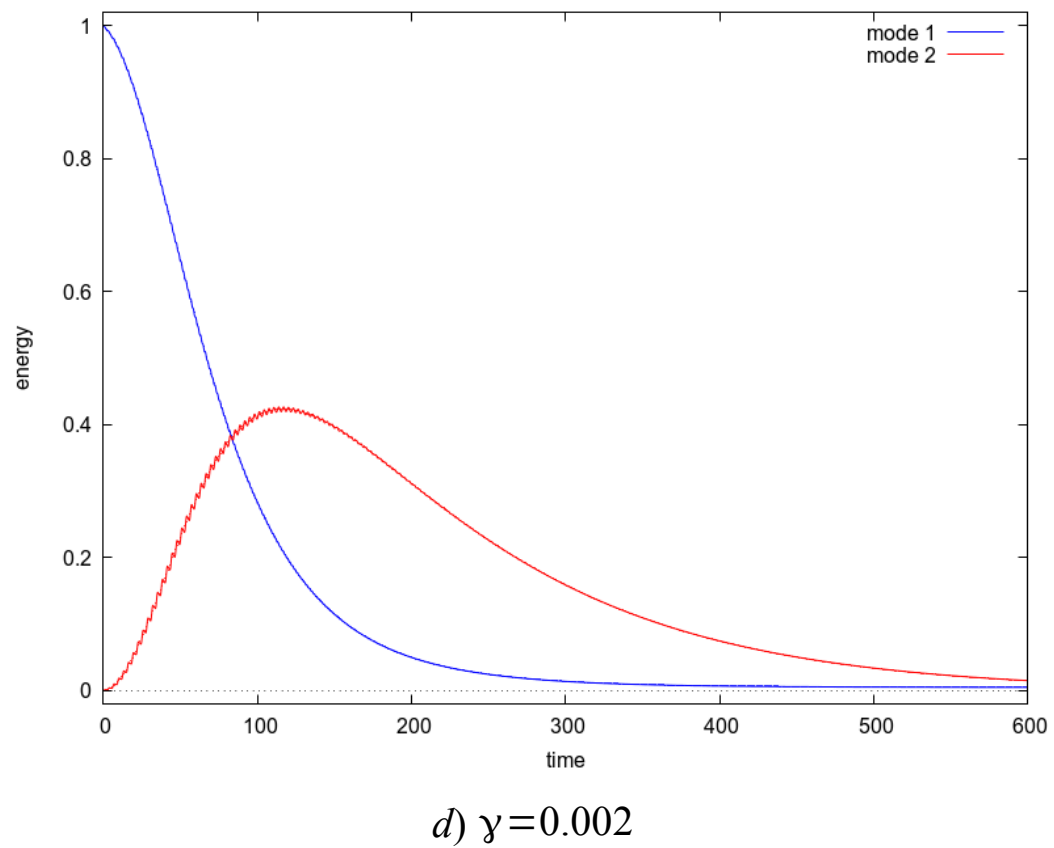

Fig. 2. Energy exchange between the two leading modes. Solid curves - numerical solution, dotted curves - asymptotic solution. 


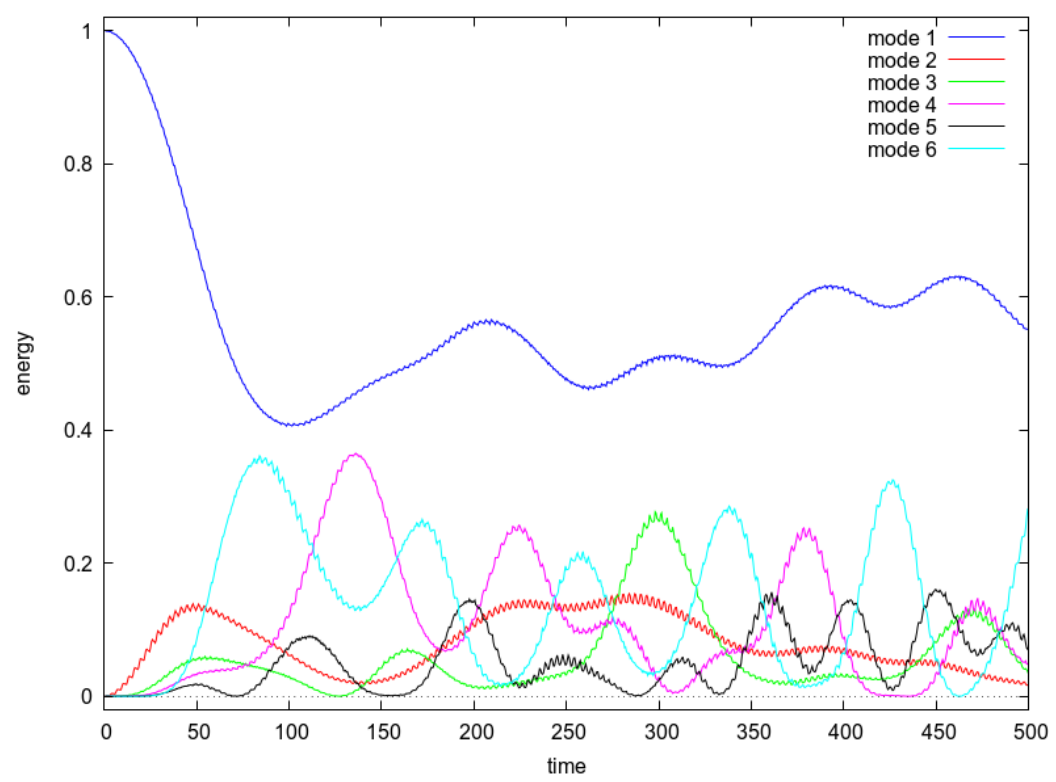

a) $\gamma=0$ (non-dissipative case)

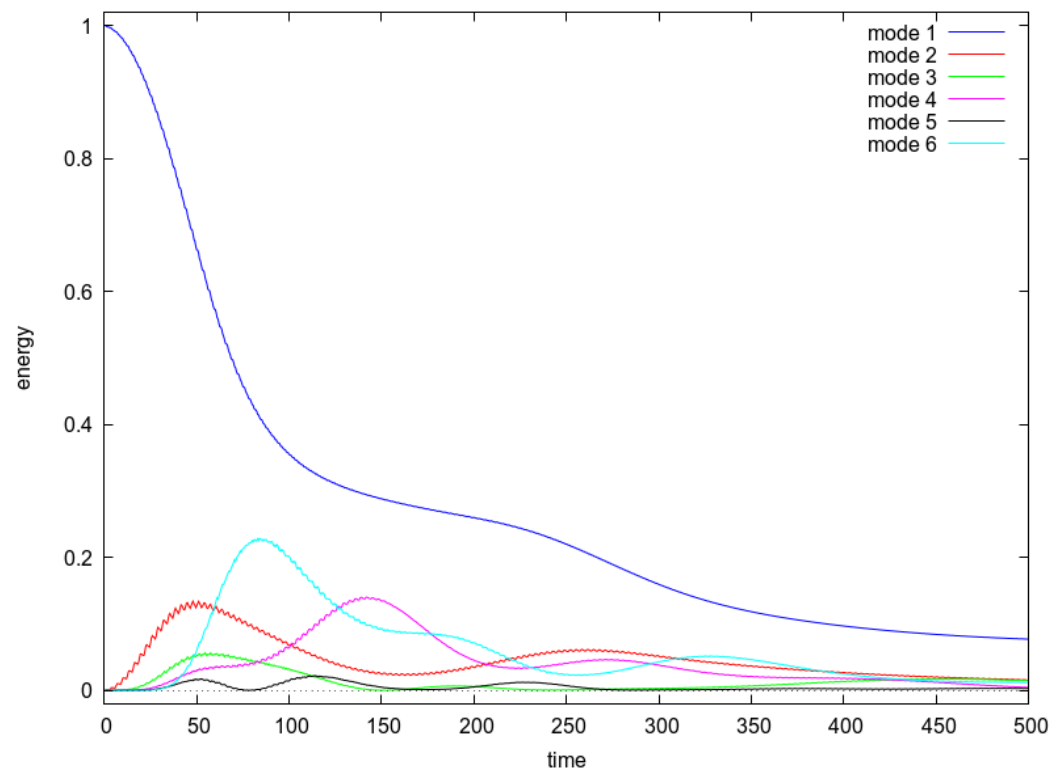

b) $\gamma=0.0005$ 


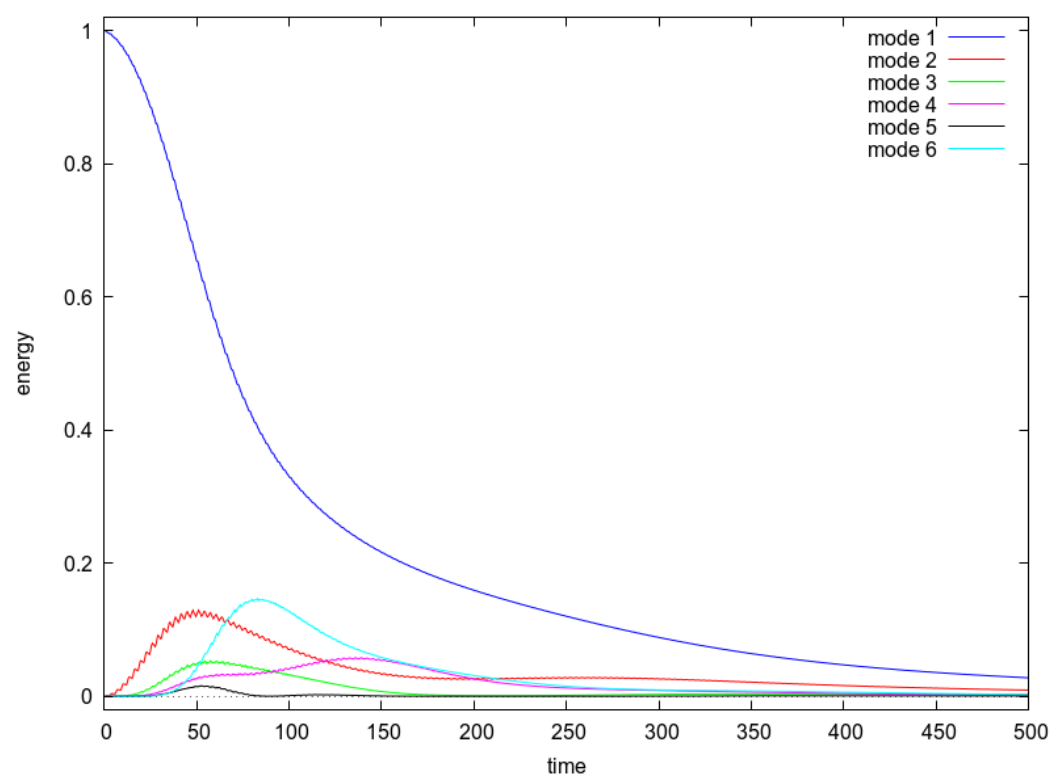

c) $\gamma=0.001$

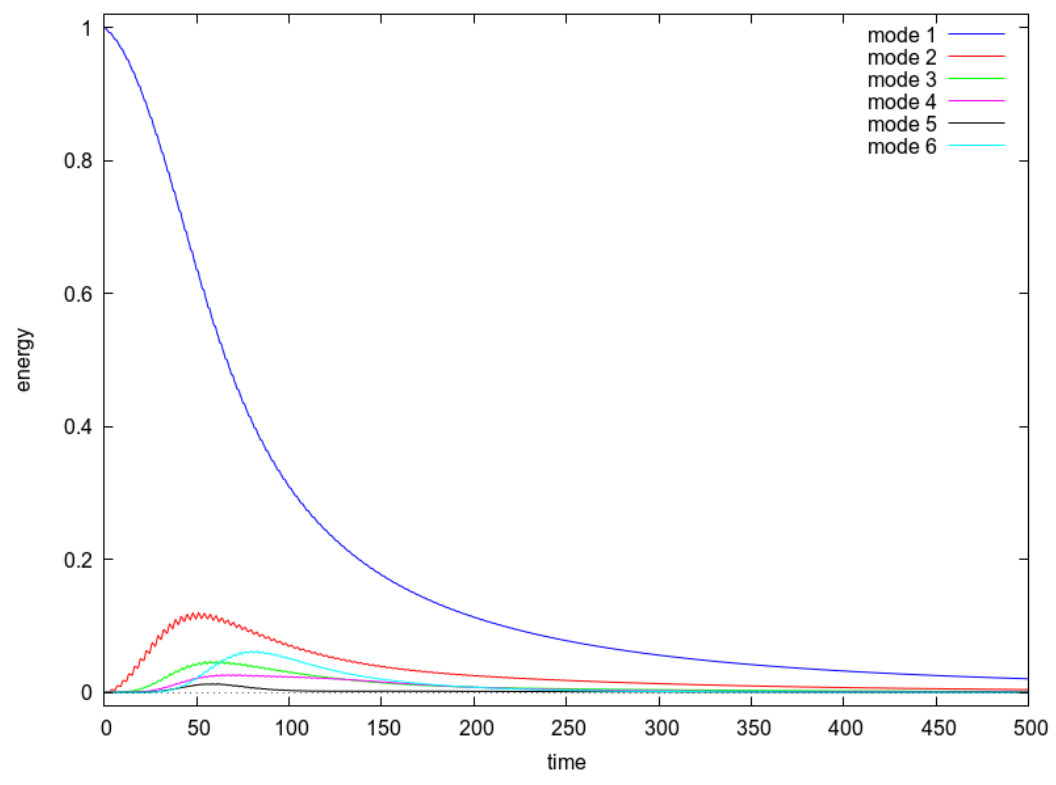

d) $\gamma=0.002$

Fig. 3. Energy transfers between the six leading modes.

\section{Conclusions}

We studied natural vibrations of a heterogeneous solid consisting of periodically repeated layers of two different materials. The viscoelastic properties of the components were represented by the Kelvin-Voigt model. Geometric non-linearity was introduced by the Cauchy-Green strain tensor and physical non-linearity was modelled employing the Murnaghan elastic potential. The 
characteristic size of the microstructure (e.g., a length of the unit cell) was supposed to be essentially smaller than the scale of the macroscopic problem (e.g., spatial periods of the vibration modes). Then a homogenised dynamical equation was proposed to describe the dynamic properties of the medium on the macro level. We emphasise that all the coefficients of the homogenised model were determined explicitly encapsulating information about the properties of the microstructure. This gives an advantage before many phenomenological approaches, where the material parameters are usually left unknown.

Using a Fourier series expansion, the input continuous problem was discretised and reduced to an infinite system of non-linear ordinary differential equations. An asymptotic solution was developed by the method of multiple time scales and numerical simulations were performed by the fourth-order Runge-Kutta method. The obtained numerical and analytical results exhibit a good agreement.

We studied the interplay between the effects of non-linearity and dissipation. In the case of a purely elastic material, an infinite number of the vibration modes may be coupled by non-linear internal resonances. This results in periodic energy transfers between different modes and in a selfgeneration of higher-order modes. Hence, truncation to the modes having non-zero initial energy is prohibited.

If the viscosity increases, the dissipation suppresses the influence of non-linearity. The energy transfers between the vibration modes are restricted and, on a large time scale, the modes coupling vanishes. The attenuation coefficient of the $n$th mode is proportional to $n^{2}$, so the internal dissipation exhibits a quadratic growth with the increase of the mode number. In such a case, truncation of the original infinite system to only a few leading order modes can provide a reasonable approximation.

\section{Acknowledgement}

This work has received funding from the European Union's Horizon 2020 research and innovation programme under Marie Sklodowska-Curie grant agreement no. 655177 (for V.V. Danishevskyy).

\section{References}


[1] V.I. Erofeev, Wave Processes in Solids with Microstructure, World Scientific, Singapore, 2003.

[2] A.V. Porubov, Amplification of Nonlinear Strain Waves in Solids, World Scientific, Singapore, 2003.

[3] Yu.V. Belashov, S.V. Vladimirov, Solitary Waves in Dispersive Complex Media, Springer, Berlin, Heidelberg, 2005.

[4] V.P. Maslov, P.P. Mosolov, Nonlinear Wave Equations Perturbed by Viscous Terms, de Gruyter, Berlin, New York, 2000.

[5] W. Flügge, Viscoelasticity, Springer, New York, 1975.

[6] V.A. Pal'mov. Propagation of vibrations in a nonlinear dissipative medium, Journal of Applied Mathematics and Mechanics 31 (1967) 763-769.

[7] V.A. Pal'mov, Vibrations of Elasto-Plastic Bodies, Springer, Berlin, Heidelberg, 2014.

[8] E. Varley, T.G. Rogers, The propagation of high frequency, finite acceleration pulses and shocks in viscoelastic materials, Proceedings of the Royal Society A 296 (1967) 498-518.

[9] R.B. Seymour, E. Varley, High frequency, periodic disturbances in dissipative systems. I. Small amplitude, finite rate theory, Proceedings of the Royal Society A 314 (1970) 387-415.

[10] M.P. Mortell, E. Varley, Finite amplitude waves in bounded media: nonlinear free vibrations of an elastic panel, Proceedings of the Royal Society A 318 (1970) 169-196.

[11] M.P. Mortell, R.B. Seymour, Pulse propagation in a nonlinear viscoelastic rod of finite length, SIAM Journal on Applied Mathematics 22 (1972) 209-224.

[12] P. Ribeiro, M. Petyt, Non-linear vibration of beams with internal resonances by the hierarchical finite-element method, Journal of Sound and Vibration 224 (1999) 591-624.

[13] A.H. Nayfeh, D.T. Mook, Nonlinear Oscillations, Wiley, New York, 1979.

[14] G.J. Boertjens, W.T. van Horssen, On mode interactions for a weakly nonlinear beam equation, Nonlinear Dynamics 17 (1998) 23-40.

[15] G.J. Boertjens, W.T. van Horssen, An asymptotic theory for a weakly nonlinear beam equation with a quadratic perturbation, SIAM Journal on Applied Mathematics 60 (2000) 602-632.

[16] A.I. Manevich, L.I. Manevitch, The Mechanics of Nonlinear Systems with Internal Resonances, World Scientific, Singapore, 2005.

[17] H. Arvin, F. Bakhtiari-Nejad, Nonlinear modal interaction in rotating composite Timoshenko beams, Composite Structures 96 (2013) 121-134.

[18] I.V. Andrianov, V.V. Danishevskyy, B. Markert, Nonlinear vibrations and mode interactions for a continuous rod with microstructure, Journal of Sound and Vibration 351 (2015) 268281. 
[19] I.V. Andrianov, J. Awrejcewicz, V.V. Danishevskyy, B. Markert, Influence of geometric and physical nonlinearities on the internal resonances of a finite continuous rod with a microstructure, Journal of Sound and Vibration 386 (2017) 359-371.

[20] T.P. Weissert, The Genesis of Simulations in Dynamics - Pursuing the Fermi-Pasta-Ulam Problem, Springer, Berlin, 1997.

[21] M. Maldovan, Sound and heat revolution in phononics, Nature 503 ( 2013) 209-217.

[22] M.I. Hussein, M.J. Leamy, M. Ruzzene, Dynamics of phononic materials and structures: historical origins, recent progress, and future outlook. Applied Mechanics Reviews 66 (2014) 040802.

[23] B. Liang, B. Yuan, J.C. Cheng, Acoustic diode: rectification of acoustic energy flux in onedimensional systems, Physical Review Letters 103 (2009) 104301.

[24] M.I. Hussein, G.M. Hulbert, R.A. Scott, Dispersive elastodynamics of 1D banded materials and structures: analysis, Journal of Sound and Vibration 289 (2006) 779-806.

[25] A.I. Lur'e, Nonlinear Theory of Elasticity, North-Holland, Amsterdam, 1990.

[26] F.D. Murnaghan, Finite Deformation of an Elastic Solid, Wiley, New York, 1951.

[27] I.V. Andrianov, V.V. Danishevs'kyy, O.I. Ryzhkov, D. Weichert, Dynamic homogenization and wave propagation in a nonlinear 1D composite material, Wave Motion 50 (2013) 271-281.

[28] N.S. Bakhvalov, G.P. Panasenko, Averaging Processes in Periodic Media, Mathematical Problems in Mechanics of Composite Materials. Kluwer, Dordrecht, 1989.

[29] G.M. Chechin, V.R Sakhnenko, Interactions between normal modes in nonlinear dynamical systems with discrete symmetry. Exact results, Physica D 117 (1998) 43-76. 